\title{
Biochemical markers of nutritional status and childhood malaria severity in Cameroon
}

\author{
Joël Bertrand Pankoui Mfonkeu ${ }^{1}$, Inocent Gouado ${ }^{1 *}$, Honoré Fotso Kuaté ${ }^{2}$, Odile Zambou ${ }^{3}$, \\ Valéry Combes ${ }^{4}$, Georges Emile Raymond Grau ${ }^{4}$ and Paul Henri Amvam Zollo ${ }^{1}$ \\ ${ }^{1}$ Department of Biochemistry, Faculty of Science, University of Douala, PO Box 24157, Douala, Cameroon \\ ${ }^{2}$ Laboratory Service, Laquintinie Hospital, Douala, Cameroon \\ ${ }^{3}$ Paediatric Service, Deido District Hospital, Douala, Cameroon \\ ${ }^{4}$ Vascular Immuno-pathology Unit, Faculty of Medicine, University of Sydney, PO Box 24157, Sydney, NSW 2042, Australia
}

(Received 10 June 2009 - Revised 11 March 2010 - Accepted 22 March 2010 - First published online 9 July 2010)

To investigate the part played by undernutrition in malaria severity, some biomarkers of nutritional status were assessed in children with severe malarial anaemia (MA) and cerebral malaria (CM) in comparison with healthy children or those with uncomplicated malaria. Undernutrition was assessed using the weight-for-age $Z$ score (WAZ). Retinol was determined by HPLC; lipid profile, $\mathrm{Ca}, \mathrm{Mg}$ and albumin were determined by spectrophotometry. Severe and moderate undernutritions were more prevalent in children with MA and those with the combined symptoms of CM and MA, but not in those with CM alone. Some perturbations were noticed in the lipid profile, but most of the values remained within the normal ranges. The risk of vitamin A deficiency, as assessed by plasma retinol concentration, was noteworthy in children with severe malaria: $0.48 \times 10^{-6}$ and $0.50 \times 10^{-6} \mathrm{~mol} / \mathrm{l}$, respectively, in children with MA and CM (reference value: $>0.7 \times 10^{-6} \mathrm{~mol} / \mathrm{l}$ ). A significant difference was obtained for retinol values after an ANOVA of all the groups $(P=0.0029)$, with the value in the MA group being significantly low than that in the control group $(P<0 \cdot 05)$; likewise, a significant difference was obtained after comparison of all the groups for Mg and albumin $(P=0.0064$ and 0.0082 , respectively). Despite their low number $(n 6)$, fatal cases of $\mathrm{CM}$ had a normal mean WAZ on admission, but low values of retinol, albumin and HDL:LDL ratio. Despite these associations, undernutrition itself did not appear to be a primary factor associated with fatal outcome.

\section{Undernutrition: Malaria severity: Cameroonian children}

Malaria is one of the most common causes of morbidity and mortality in sub-Saharan Africa; each year, an estimated 1-2.8 million persons, mostly children, die of malaria. Approximately, 1-2\% of clinical cases of malaria in African children lead to cerebral malaria (CM), which is a severe form of the disease ${ }^{(1)}$. CM and malarial anaemia (MA) are considered to be the major complications of the disease. The understanding of events leading to these severe forms is crucial in the fight against malaria, and more precisely, to prevent the occurrences of these severe forms ${ }^{(2,3)}$.

Malnutrition may contribute to every second death (53\%) associated with infectious diseases among children under 5 years of age in developing countries ${ }^{(4,5)}$. As characteristics of malnutrition, deficiencies and disturbances in some nutritional parameters have been studied in relation to malaria.

Vitamin A deficiency as estimated by the measurement of plasma retinol concentration has been shown to be prevalent among malaria-infected individuals ${ }^{(6-8)}$, and was attributed to inflammatory response and redistribution of vitamin A into extravascular spaces to allow increased bioavailability to the tissues ${ }^{(9)}$. Also, as severe malaria shares many features with sepsis syndrome, electrolyte derangements (including $\mathrm{Ca}$, $\mathrm{Mg}, \mathrm{P}$ and $\mathrm{K}$ ), commonly associated with sepsis, have been postulated to complicate severe malaria ${ }^{(10)}$. However, these nutrient deficiencies and derangements in the particular context of the two most severe forms of malaria (severe MA and $\mathrm{CM}$ ) are yet to be largely addressed. Their investigations could bring more insight into the features of these deadly forms of malaria.

In addition, more is required to be understood about changes in albumin and its associated lipid parameters in relation to malaria ${ }^{(11)}$. Hypocholesterolaemia and hypertriacylglycerolaemia were observed in settings of both uncomplicated and complicated malaria ${ }^{(12,13)}$. Population studies of common lipid parameters indicate that cholesterol values are currently lower in Africa, where malaria is endemic, than in many other parts of the world ${ }^{(14)}$. Furthermore, it has been shown that in vitro serum albumin and its associated fatty acids are essential for intraerythrocytic development and cell cycle progression of Plasmodium falciparum ${ }^{(15)}$. Several studies have shown that parasites induce significant changes in lipid parameters ${ }^{(16,17)}$. In human subjects, cholesterol is 
synthesised in the liver, which is incidentally the first hosting organ for the malaria parasites after infecting the human host and one of the major multiplication sites for the Plasmodium ${ }^{(13,18,19)}$. However, the magnitude of these interactions in relation to the severity of malaria in human and the status of common lipid parameters and albumin in children with the most severe forms of malaria need clarification.

Here, we have investigated undernutrition and associated plasma lipid profile, albumin, retinol, $\mathrm{Ca}$ and $\mathrm{Mg}$ in children with severe malaria (severe $\mathrm{MA}$ and $\mathrm{CM}$ ) in comparison with healthy children or those with uncomplicated malaria (UCM). The objective of the present study was to assess the part played by these biomarkers of nutritional status in relation to malaria severity and the occurrence of fatalities.

\section{Methods}

This hospital-based study was conducted from January to December 2007 on 225 consecutive paediatric admissions of malaria cases and forty-five children who had come for vaccination or counselling (control) in four hospitals in Douala, namely Laquintinie hospital (Akwa), Deido district hospital (Deido), Palmier district hospital (Cité des Palmiers) and Emilie Saker pediatric center (Akwa), which are all situated in populous areas of the town. Children with diarrhoea, non-malarial infections and HIV were excluded. Finally, children who met the study inclusion criteria were recruited after informed consent, and at a later time, they were allocated to the different malaria severity groups: UCM, MA, CM, and the combined symptoms of CM and MA (CM \& MA), following the 2000 WHO criteria ${ }^{(20)}$. The definitions of the different categories of patients recruited are described below.

Baseline characteristics and clinical outcome of these children are described in detail elsewhere ${ }^{(21)}$. Briefly, anthropometric data, information on the use of impregnated bed net to prevent malaria, significant medical history and recent drug usage were obtained; the latter was recorded due to the availability of antimalarials for prophylaxis/medication in our setting, but which are not always properly used. Clinical data such as temperature and blood pressure (when possible) were recorded every $6 \mathrm{~h}$ during hospitalisation. Complete physical examinations, including neurological status according to the Blantyre coma scale and prostration assessment (defined as inability to sit unassisted in a child who can normally do so or inability to drink in a child who cannot normally sit up), were performed. The anthropometric data were used to investigate malnutrition.

Fasting blood sample was extracted by venous puncture, and it was collected into citrated and EDTA sterile tubes on admission to the hospital (and before any treatment). A drop was used for thick blood film preparation. The plasma was obtained by centrifugation of the citrated blood sample at $1000 \mathrm{~g}$ for $10 \mathrm{~min}$ using a clinical centrifuge (Sigma Bioblock Scientific, Illkirch, France), and was used to investigate lipid profile and retinol. Haematological parameters (including leucocyte, erythrocyte and platelet counts, as well as $\mathrm{Hb}$ level and haematocrit) were determined using an automated coulter (Celly 70, Bordeaux, France) with the blood collected in an EDTA tube. Another fasting blood sample was collected at discharge in severe malaria patients, and processed as the admission sample.

\section{Definition of categories}

MA was taken to be an $\mathrm{Hb}$ concentration $<80 \mathrm{~g} / \mathrm{l}$ or a haematocrit $<18 \%$ in a patient who had a positive malaria smear. CM was diagnosed if a patient with a positive smear for malaria presented with impaired consciousness as measured using the Blantyre coma score $\leq 2$ (range: $0-5$ ) and a clear and normal cerebrospinal fluid (glucose level between $0 \cdot 5$ and $0.8 \mathrm{~g} / 1$, and total protein level between 0.15 and $0.45 \mathrm{~g} / \mathrm{l})$. The coma score was determined as described by Molyneux et al. ${ }^{(22)}$ for all comatose patients. Children without any of the above-mentioned symptoms, but presenting the usual malaria symptoms and a positive malaria smear, were classified as UCM patients.

\section{Malaria diagnosis}

Blood was spotted on the slide, and thick films were prepared in duplicate. They were stained with $10 \%$ Giemsa solution for $20 \mathrm{~min}$, and were then allowed to dry ${ }^{(23)}$. The parasites were counted with a microscope (Motic Deutschland $\mathrm{GmbH}$, Wetzlar, Germany) using the thick films on the basis of the number of parasites per 200 leucocytes counted; this was converted to the number of parasites per microlitre of blood, taking into account the leucocyte count of the subject. For control subjects, in addition to the thick blood film, a more sensitive antigenic test was carried out to detect P. falciparum-specific histidine-rich protein 2 using ParaHit dipstick (Span Diagnostics Limited, Surat, India).

\section{Malnutrition investigation: weight-for-age $\mathrm{Z}$ score determination}

Weight-for-age $Z$ score (WAZ) was used to assess wasting as an indicator of undernutrition because of its availability and its ability to evaluate recent acute undernutrition ${ }^{(24)}$.

Patients' WAZ were measured, and in accordance with the anthropometric measurements at the moment of hospitalisation, each patient's nutritional status was evaluated. This evaluation took into account the patients' weight, as well as the median weight of control patients of the same age and sex. The calculations were done according to the following formula ${ }^{(25)}$ :

$$
\begin{aligned}
\mathrm{WAZ}= & (\text { observed weight } \\
& - \text { median weight }(\text { same age and sex })) / \text { standard deviation. }
\end{aligned}
$$

\section{Retinol analysis}

Retinol analysis was done by a method initially described by Bieri et al. ${ }^{(26)}$. Briefly, $200 \mu$ l of plasma were introduced into a tube, and $500 \mu \mathrm{l}$ of retinyl acetate (at a concentration of $100 \mu \mathrm{g} / \mathrm{ml}$; Puritan's Pride, Bohemia, NY, USA) in ethanol were added as internal standard, and homogenised using a vortex; then, $1 \mathrm{ml}$ of hexane was added, homogenised using a vortex and centrifuged at $450 \mathrm{~g}$ for $5 \mathrm{~min}$ at $-5^{\circ} \mathrm{C}$. The supernatant $(500 \mu \mathrm{l})$ was removed using a pipette (single use) and introduced into a second tube, and the residue was extracted again using $1 \mathrm{ml}$ of hexane. All the hexane extracts that were obtained were pooled together and evaporated under nitrogen. 
The residue obtained was dispersed into $200 \mu$ l of methanol; after passing through a sonicator, $60 \mu \mathrm{l}$ were injected into the HPLC system (supelcosil LC-18; Supelco, Bellefonte, PA, USA) comprising a column (supelco 58298; $250 \times 4.6 \mathrm{~mm}$ diameter), $5 \mu \mathrm{m}$ particle size, a precolumn, a pump (Altech 426), an integrator (HP 3395) and a detector (Diode Array detector; Hewlett-Packard, Dublin, Ireland), Linear UVIS 200. The elution was done with a mobile phase made of methanolacetonitrile-water (93:5:2, by vol.) at a speed of $2 \mathrm{ml}$ per min, wave length of detection, $325 \mathrm{~nm}$, range, 0.01 absorbance units full scale (AUFS); rise time, $0 \cdot 3 \mathrm{~s}$. The analysis was done using yellow light to prevent the destruction of vitamin A.

Addition of the exact quantity of the internal standard to a known volume of the sample was done for the calculation of the vitamin A in plasma, and that concentration was calculated from peak heights, adjusted by the internal standard response.

\section{Lipid profile, albumin, calcium and magnesium analysis}

Lipid profile was investigated by the determination of the plasma total cholesterol, HDL cholesterol, LDL cholesterol, TAG and the HDL:LDL cholesterol ratio. Total cholesterol, HDL, TAG, as well as albumin, $\mathrm{Ca}$ and $\mathrm{Mg}$ were all determined by spectrophotometry using commercial kits. More precisely, total cholesterol was determined using cholesterol oxidase and peroxidase (SGM, Rome, Italy); HDL was separated with polyethylene glycol and determined as the total cholesterol (SGM); TAG were determined using lipoprotein lipase, glycerolkinase and 4-aminophenazone, respectively (SGM); albumin, $\mathrm{Ca}$ and $\mathrm{Mg}$ were determined using bromocresol green (Hospitex Diagnostics, Firenze, Italy), ortho-cresolphthalein (Human, Wiesbaden, Germany) and xylidyl blue (Human, Wiesbaden, Germany), respectively. LDL was calculated using the Friedewald formula ${ }^{(27)}$ :

$$
\operatorname{LDL}(\mathrm{g} / \mathrm{l})=\text { total cholesterol }-\mathrm{HDL}-(\mathrm{TAG}) / 5 \text {. }
$$

\section{Quality control}

For retinol analysis, the inter-assay and intra-assay CV for a quality-control serum $\left(2.39 \times 10^{-6} \mathrm{~mol} / \mathrm{l}\right)$ included in duplicate with each run were $12.6 \%$ (n 33 pairs) and $5.8 \%$ (n 24 pairs), respectively. For albumin, $\mathrm{Ca}, \mathrm{Mg}$ and all the parameters of lipid profile, intra-assay precision tests were done on thirty samples for each control (low - normal high), and inter-assay precision tests were done on fifteen samples for each control (low - normal - high) for $3 \mathrm{~d}$. The $\mathrm{CV}$ obtained were all less than $5 \%$ (range: $1 \cdot 47-3 \cdot 35 \%$ ).

\section{Statistical methods}

Data were analysed using GraphPad Prism version 5.00 for Windows (GraphPad Software, San Diego, CA, USA). Variables were log transformed and compared with Student's $t$ test (two categories) or ANOVA (several categories). Paired $t$ test was used when the two sets of data were from the same individual, for example, admission and discharge data of the same subject. Differences between the proportions (prevalence) calculated were tested using $\chi^{2}$ tests. Pearson's correlation coefficients $(r)$ were used as the measurement of correlation. A $P<0.05$ was considered significant.

\section{Ethical considerations}

The present study was conducted according to the guidelines laid down in the Declaration of Helsinki, and all procedures involving human subjects were approved by the Cameroon National Ethics Committee (PO BOX 1937, Yaoundé, Cameroon) and the Delegation of the Cameroonian ministry of public health covering the town of Douala according to the authorisation no. 086/CNE/DNM/07. Verbal informed consent was obtained from all subjects, witnessed by the physician in charge, and formally recorded.

\section{Results}

Of the 225 children recruited, 94 children had UCM (age range: 6-168 months), 73 children had MA (7-156 months), 45 children had CM (6-134 months) and 13 children had the combined symptoms of MA and CM (9-96 months). Also, forty-five controls (6-156 months) were recruited from those who had come for vaccination and counselling. Table 1 presents an overview of anthropometric and clinical characteristics of subjects in the different groups. It shows that CM patients were the youngest, with the average age being $26 \cdot 1$ months in the group. Their mean baseline temperature was also the highest $\left(39 \cdot 8^{\circ} \mathrm{C}\right)$, and the mean parasite level in the group was also high (138007 parasites/ $\mu 1)$. Obviously, groups with anaemia had low levels of $\mathrm{Hb}(57.2$ and $61.5 \mathrm{~g} / \mathrm{l}$ in the MA and CM \& MA groups, respectively). Furthermore, six patients from the CM group died in the hospital $(13.3 \%$ of the CM patients). These were the only fatal cases recorded during our recruitments.

\section{Malnutrition prevalence}

WAZ are typically categorised in nutritional terms as mild $(-1.01$ to $-2.00 \mathrm{SD})$, moderate $(-2.01$ to $-3.0 \mathrm{SD})$ or severe $(<-3.0 \mathrm{SD})^{(24)}$. Table 2 presents the prevalence of malnutrition in the different groups.

From this table, we can observe that severe and moderate undernutritions were more prevalent in children with the combined symptoms of MA and CM (15.38 and 23.08\%, respectively) and in children with MA (6.85 and 16.44\%, respectively). They were the only groups with prevalence statistically different from that in the control group $(P<0.001)$. When we combined these two groups (MA and CM \& MA groups), the prevalence was even statistically different from that in the UCM and CM groups $(P<0 \cdot 001)$. However, mild undernutrition was more prevalent in children with CM (46.67\%).

\section{Biochemical markers of nutrition and malaria severity}

Table 3 presents the biochemical markers of nutritional status investigated on admission in the different groups. Mean total cholesterol was low in two groups with severe malaria ( 0.91 and $0.88 \mathrm{~g} / \mathrm{l}$, respectively, in MA and $\mathrm{CM}$ patients); particularly, the mean level in the CM group was significantly 
Table 1. Anthropometric, clinical and parasitological characteristics

(Mean values with their standard errors)

\begin{tabular}{|c|c|c|c|c|c|c|c|c|c|c|}
\hline \multirow[b]{2}{*}{ Parameters } & \multicolumn{2}{|c|}{ Controls } & \multicolumn{2}{|c|}{ UCM } & \multicolumn{2}{|c|}{ MA } & \multicolumn{2}{|c|}{$\mathrm{CM}$} & \multicolumn{2}{|c|}{$C M \& M A$} \\
\hline & Mean & SE & Mean & SE & Mean & SE & Mean & SE & Mean & SE \\
\hline$n$ & \multicolumn{2}{|c|}{45} & \multicolumn{2}{|c|}{94} & \multicolumn{2}{|c|}{73} & \multicolumn{2}{|c|}{45} & \multicolumn{2}{|c|}{13} \\
\hline Number of males & \multicolumn{2}{|c|}{14} & \multicolumn{2}{|c|}{58} & \multicolumn{2}{|c|}{43} & \multicolumn{2}{|c|}{22} & \multicolumn{2}{|c|}{7} \\
\hline Age (months) & $59 \cdot 07$ & $8 \cdot 82$ & 41.56 & $5 \cdot 12$ & 49.93 & $5 \cdot 71$ & $26 \cdot 13^{\star}$ & $2 \cdot 41$ & $36 \cdot 70$ & 8.98 \\
\hline Weight (kg) & 19.05 & 1.47 & $15 \cdot 68$ & 1.22 & $15 \cdot 63$ & 1.09 & $12 \cdot 18^{\star}$ & 0.65 & $13 \cdot 25$ & 1.27 \\
\hline Temperature $\left({ }^{\circ} \mathrm{C}\right)$ & 37.19 & 0.04 & $38.91^{*}$ & 0.09 & $38 \cdot 63^{*}$ & 0.10 & $39.83^{*}$ & $0 \cdot 16$ & $39.57^{*}$ & 0.33 \\
\hline FRT (h) & NA & NA & 34.40 & $2 \cdot 68$ & $39 \cdot 17$ & 4.08 & $58.11 \dagger$ & 5.58 & $96.00 \dagger$ & 6.41 \\
\hline BCS & 5.00 & 0.00 & 4.75 & 0.06 & 4.42 & $0 \cdot 10$ & $1.66^{*}$ & $0 \cdot 10$ & $1.70^{*}$ & 0.15 \\
\hline CRT (h) & NA & NA & NA & NA & NA & NA & $14 \cdot 10$ & 1.40 & $27 \cdot 00$ & $3 \cdot 71$ \\
\hline PRT(h) & NA & NA & $19 \cdot 05$ & $2 \cdot 80$ & $46.06 \dagger$ & $4 \cdot 24$ & $52 \cdot 67 \dagger$ & 4.67 & $90.00 \dagger$ & $12 \cdot 63$ \\
\hline $\mathrm{Hb}(\mathrm{g} / \mathrm{l})$ & 124.7 & 8.2 & $111 \cdot 6^{*}$ & $13 \cdot 2$ & $57 \cdot 2^{*}$ & 13.5 & $103 \cdot 7^{\star}$ & $10 \cdot 4$ & $61.5^{\star}$ & $12 \cdot 7$ \\
\hline Parasites (per $\mu$ l whole blood) & NA & NA & 14518 & 2443 & $67345 \dagger$ & 13512 & $138007 \dagger$ & 28183 & $141000 \dagger$ & 50784 \\
\hline
\end{tabular}

UCM, uncomplicated malaria; MA, malarial anaemia; CM, cerebral malaria; $n$, number of subjects; FRT, fever resolution time; BCS, Blantyre coma score ${ }^{(22)}$; CRT, coma resolution time; PRT, prostration resolution time; NA, not applicable.

${ }^{*}$ Value in this group was statistically different from the value in the control group $(P<0.05)$

† Value in this group was statistically different from the value in the UCM group $(P<0.05)$.

lower than the mean level in the control group $(1.19 \mathrm{~g} / \mathrm{l})$, $P<0.05$. In fact, there was a significant difference between the mean levels of total cholesterol in all the groups, $P=0.0049$.

The mean values of retinol in the different groups were all low in comparison with the reference value of $0.7 \times 10^{-6} \mathrm{~mol} / \mathrm{l}$, and the mean values in the patient groups were generally significantly lower than the mean value in the control group, $P=0.0029$. This was more remarkable in children with MA $\left(0.48 \times 10^{-6} \mathrm{~mol} / \mathrm{l}\right), P<0.05$. It is to be noted that the volumes of most of the samples from children with the combined symptoms of $\mathrm{CM}$ and MA were not sufficient for the retinol analysis.

The mean values of $\mathrm{Mg}$ in the UCM, CM and CM \& MA groups were individually significantly low $(P<0.05)$ than the mean value in the control group. We obtained mean values of $0.82 \times 10^{-3}, 0.61 \times 10^{-3}, 0.70 \times 10^{-3}, 0.60 \times 10^{-3}$ and $0.48 \times 10^{-3} \mathrm{~mol} / \mathrm{l}$, respectively, in the control, UCM, MA, CM and CM \& MA groups; $P=0.0064$.

Mean values of plasma albumin were $36 \cdot 80,35 \cdot 10,30 \cdot 30$, 28.40 and $27.50 \mathrm{~g} / \mathrm{l}$ in the control, UCM, MA, CM and CM $\&$ MA groups, respectively; $P=0.0082$. Table 3 shows that the mean value in the $\mathrm{CM}$ group was significantly low than the mean value in the control group $(P<0 \cdot 05)$.

We calculated correlations between $\mathrm{Ca}$ and albumin values. Table 3 shows a positive and significant correlation in the control group $(r 0.4473, P=0.0321)$, but non-significantly in all the other groups.
Table 4 presents the values of these biochemical markers of nutritional status on admission in comparison with values at discharge. It shows that almost all the low values on admission increased at discharge, except TAG values, which decreased in the MA group $(P=0.05)$, and $\mathrm{Ca}$ values, which remained low in both the groups (CM and MA groups), as no statistical differences were found.

\section{Biomarkers of malnutrition and fatal outcome}

As stated earlier, six CM patients died in the hospital. Despite their low number, we found that mean value of retinol was significantly lower in those who died than in those who survived $\left(0.24 \times 10^{-6}\right.$ and $0.54 \times 10^{-6} \mathrm{~mol} / \mathrm{l}$ in fatal cases and survivors, respectively; $P=0 \cdot 006$ ). Some differences were also noticed for other markers, even though no statistical significance was found. The mean value of the WAZ in children who died of $\mathrm{CM}$ was $-0 \cdot 80$. This value was above -1 , and is typically categorised in nutritional terms as normal.

\section{Discussion}

In the present study, the highest prevalence of severe undernutrition was not found in $\mathrm{CM}$ patients, and even severe undernutrition was not observed in these patients, but it was rather observed in MA patients. This is in line with findings in a study of Tanzanian children with severe anaemia who were frequently asymptomatic or showed non-specific

Table 2. Malnutrition prevalence as assessed by weight-for-age $Z$ score (WAZ)

\begin{tabular}{|c|c|c|c|c|c|}
\hline Prevalence $(\%)^{*}$ & Controls ( $n$ 45) & UCM $(n 94) \dagger$ & MA $(n 73) \ddagger$ & $\mathrm{CM}+(n 45)$ & CM \& MA $(n 13)$ \\
\hline Severe undernutrition $(W A Z \leq-3)$ & 0 & $2 \cdot 12$ & 6.85 & 0 & $15 \cdot 38$ \\
\hline Moderate undernutrition $(-2.99 \leq \mathrm{WAZ} \leq-2)$ & 0 & $5 \cdot 32$ & $16 \cdot 44$ & $6 \cdot 66$ & 23.08 \\
\hline Mild undernutrition $(-1.99 \leq \mathrm{WAZ} \leq-1)$ & $15 \cdot 56$ & $12 \cdot 77$ & $26 \cdot 03$ & $46 \cdot 67$ & 7.69 \\
\hline No undernutrition (WAZ $>-1)$ & 84.44 & $79 \cdot 79$ & $50 \cdot 68$ & $46 \cdot 67$ & 53.85 \\
\hline
\end{tabular}

UCM, uncomplicated malaria; MA, malarial anaemia; CM, cerebral malaria.

${ }^{*}$ When we combined the MA and CM \& MA groups, the prevalence of the combined group was also statistically different from that of the control, UCM and CM groups; $P<0.001$ ( $\chi^{2}$ test).

†Prevalence in this group was not statistically different from the prevalence in the control group; $P>0.05\left(\chi^{2}\right.$ test)

$\ddagger$ Prevalence in this group was statistically different from the prevalence in the control group; $P<0.001\left(\chi^{2}\right.$ test). 
Table 3. Nutritional markers on admission

(Mean values with their standard errors)

\begin{tabular}{|c|c|c|c|c|c|c|c|c|c|c|c|}
\hline \multirow[b]{2}{*}{ Parameters } & \multicolumn{2}{|c|}{ Controls ( $n$ 45) } & \multicolumn{2}{|c|}{ UCM ( $n$ 94) } & \multicolumn{2}{|c|}{ MA $(n 73)$} & \multicolumn{2}{|c|}{$\mathrm{CM}(n 45)$} & \multicolumn{2}{|c|}{$\mathrm{CM} \& \mathrm{MA}(n 13)$} & \multirow[b]{2}{*}{$P^{*}$} \\
\hline & Mean & SE & Mean & SE & Mean & SE & Mean & SE & Mean & $\mathrm{SE}$ & \\
\hline \multicolumn{12}{|l|}{ Lipid profile } \\
\hline Total cholesterol (g/l) & $1 \cdot 19$ & 0.08 & 1.05 & 0.05 & $0.91 \dagger$ & 0.06 & $0.88 \dagger$ & 0.06 & 1.24 & 0.15 & 0.0049 \\
\hline $\mathrm{HDL}(\mathrm{g} / \mathrm{l})$ & 0.40 & 0.04 & 0.42 & 0.03 & 0.30 & 0.03 & 0.34 & 0.04 & 0.40 & 0.05 & 0.3068 \\
\hline TAG $(g / l)$ & 0.52 & 0.24 & 0.49 & 0.24 & 0.61 & 0.33 & 0.57 & 0.31 & 0.51 & 0.25 & 0.3624 \\
\hline $\operatorname{LDL}(\mathrm{g} / \mathrm{l})$ & 0.67 & 0.36 & 0.48 & 0.30 & 0.48 & 0.34 & 0.40 & 0.24 & 0.60 & 0.39 & 0.0753 \\
\hline HDL:LDL ratio & 2.82 & 1.66 & $2 \cdot 12$ & 1.05 & 1.20 & $1 \cdot 16$ & 1.25 & $1 \cdot 11$ & 0.55 & 0.48 & 0.5567 \\
\hline Retinol $\left(\times 10^{-6} \mathrm{~mol} / \mathrm{l}\right) \dagger$ & 0.66 & 0.06 & 0.65 & 0.09 & $0.48 \ddagger$ & 0.06 & 0.50 & 0.06 & - & - & 0.0029 \\
\hline $\mathrm{Ca}\left(\times 10^{-3} \mathrm{~mol} / \mathrm{l}\right)$ & $1 \cdot 78$ & 0.41 & 1.62 & 0.36 & 1.85 & 0.62 & 1.62 & 0.34 & 1.86 & 0.53 & 0.4282 \\
\hline $\mathrm{Mg}\left(\times 10^{-3} \mathrm{~mol} / \mathrm{l}\right)$ & 0.82 & 0.23 & $0.61 \dagger$ & 0.23 & 0.70 & 0.28 & $0.60 \dagger$ & 0.19 & $0.48 \dagger$ & 0.21 & 0.0064 \\
\hline Albumin (g/l) & $36 \cdot 80$ & 2.00 & $35 \cdot 10$ & 1.60 & $30 \cdot 30$ & $2 \cdot 2$ & $28.40 \dagger$ & 1.30 & 27.50 & $2 \cdot 60$ & 0.0082 \\
\hline Ca/albumin correlation§ & \multicolumn{2}{|c|}{0.459} & \multicolumn{2}{|c|}{-0.02} & \multicolumn{2}{|c|}{-0.01} & \multicolumn{2}{|c|}{-0.14} & \multicolumn{2}{|c|}{-0.69} & \\
\hline
\end{tabular}

UCM, uncomplicated malaria; MA, malarial anaemia; CM, cerebral malaria.

${ }^{*} P$ values for ANOVA.

† Retinol values were not determined in the CM \& MA group because the volumes of plasma samples were not sufficient.

$\ddagger$ Mean value in this group was significantly different from the value in the control group $(P<0.05)$.

$\S$ Pearson's correlation coefficients $(r)$.

I Statistically significant correlations $(P=0.0321$, two-sided $)$.

symptoms $^{(28)}$. On the contrary, a common clinical feature of $\mathrm{CM}$ is its sudden onset and rapid deterioration. Children who were admitted with $\mathrm{CM}$ presented with a $1-3 \mathrm{~d}$ history of fever, anorexia, vomiting and sometimes coughing, unlikely to cause a serious deterioration of the nutritional profile from the start ${ }^{(2)}$. This could then explain why no CM patients in the present study suffered from severe undernutrition, and only less than $7 \%$ suffered from moderate undernutrition.

Children who are underweight are thought to have increased susceptibility to malaria for a variety of reasons, notably through a reduction in the function of the immune system $^{(29)}$. When a child is undernourished, he or she may be unable to mount an appropriate immune response to the malaria parasite due to reduction in $\mathrm{T}$ lymphocytes, impairment of antibody formation, decreased complement formation, and atrophy of thymus and other lymphoid tissues, among others $^{(30,31)}$. Although the highest risk of malarial infection is associated with the most severely underweight children, the burden of disease or death is greater for children with mild to moderate underweight status (as it was in the present study with less than $47 \%$ of the CM patients having mild undernutrition) because of the high prevalence of children with mild to moderate underweight status in many countries $^{(32)}$. Overall, contrary to previously held beliefs that the undernourished individual is an unattractive host for the parasite, a well-nourished individual seems to be capable of mounting an immune response better, and to be capable of withstanding and clearing infection ${ }^{(24)}$.

As evidence of nutritional status, nutrient profile is of paramount importance. For example, a full lipid profile can be an important part of a child's medical history and important information for a child's physician to have. The present study describes the lipid profile of children with the different symptoms of severe malaria (MA and CM patients) in comparison with those with UCM and control subjects. Total cholesterol as well as HDL and LDL cholesterols was low in children with severe malaria (both MA and CM patients); however, the values remained within their normal ranges, and may just signal a perturbation in the lipid profile. The same lipid parameters were also found to be low in a previous study

Table 4. Nutritional marker values on admission in comparison with values at discharge in severe malaria patients (Mean values with their standard errors)

\begin{tabular}{|c|c|c|c|c|c|c|c|c|c|c|}
\hline \multirow[b]{2}{*}{ Parameters* } & \multicolumn{2}{|c|}{ MA on admission } & \multicolumn{2}{|c|}{ MA at discharge } & \multirow[b]{2}{*}{$P \dagger$} & \multicolumn{2}{|c|}{$\mathrm{CM}$ on admission } & \multicolumn{2}{|c|}{$\mathrm{CM}$ at discharge } & \multirow[b]{2}{*}{$P \dagger$} \\
\hline & Mean & SE & Mean & SE & & Mean & SE & Mean & SE & \\
\hline \multicolumn{11}{|l|}{ Lipid profile } \\
\hline Total cholesterol $(\mathrm{g} / \mathrm{l})$ & 0.91 & 0.06 & 1.03 & 0.13 & $0 \cdot 16$ & 0.88 & 0.06 & 1.09 & 0.34 & 0.10 \\
\hline HDL (g/l) & 0.30 & 0.03 & 0.35 & 0.05 & 0.40 & 0.34 & 0.04 & 0.47 & 0.05 & 0.23 \\
\hline TAG $(g / l)$ & 0.61 & 0.33 & 0.36 & 0.14 & 0.05 & 0.57 & 0.31 & 0.44 & 0.21 & 0.15 \\
\hline LDL (g/l) & 0.48 & 0.34 & 0.61 & 0.40 & 0.60 & 0.40 & 0.24 & 0.53 & 0.35 & 0.18 \\
\hline HDL:LDL ratio & $1 \cdot 20$ & $1 \cdot 16$ & $1 \cdot 27$ & 1.47 & 0.79 & $1 \cdot 25$ & $1 \cdot 11$ & 1.34 & 0.96 & 0.94 \\
\hline $\mathrm{Ca} \times 10^{-3}(\mathrm{~mol} / \mathrm{l})$ & 1.85 & 0.62 & 1.74 & 0.44 & 0.54 & 1.62 & 0.34 & 1.58 & 0.40 & 0.13 \\
\hline $\mathrm{Mg} \times 10^{-3}(\mathrm{~mol} / \mathrm{l})$ & 0.70 & 0.28 & 0.77 & 0.35 & 0.22 & 0.60 & 0.19 & 0.60 & 0.20 & 0.89 \\
\hline Albumin $(\mathrm{g} / \mathrm{l})$ & $30 \cdot 30$ & $2 \cdot 20$ & 36.60 & 3.40 & 0.43 & 28.40 & 1.30 & $36 \cdot 90$ & $3 \cdot 10$ & 0.07 \\
\hline
\end{tabular}

MA, malarial anaemia; $\mathrm{CM}$, cerebral malaria.

* Data represent measured values in the same patients on admission and at discharge; retinol values are not represented because they were not determined due to insufficient volumes of plasma samples at discharge.

$\dagger P$ value of paired Student's $t$ test (two-sided). 
conducted in a selected group of $P$. falciparum-infected Cameroonians when compared with the values in healthy controls $^{(33)}$. It has also been shown that reductions in plasma total, HDL and HDL cholesterols mark an acute phase response even during minor illness ${ }^{(34)}$. However, in another study that investigated the lipid profile in patients infected with $P$. falciparum in India, an increase in all plasma lipid parameters in patients with severe malaria was found, except in $\mathrm{TAG}^{(35)}$. This difference can be explained by the pattern of patients recruited due to the level of endemicity in the study areas and the immune status of those subjects ${ }^{(20,36)}$. We also found an increase in cholesterol levels at discharge. This is in line with the results of a Gabonese study, which showed the impact of sustained parasite clearance on common lipid parameters ${ }^{(17)}$.

There are a number of ways to measure prevalence of vitamin A deficiency. Serum retinol concentrations are often used with a cut-off value $<0.70 \mu \mathrm{mol} / \mathrm{l}$. The mean levels of retinol were remarkably low in children with $\mathrm{MA}$ and $\mathrm{CM}$ (0.48 (SE 0.06) and 0.50 (SE 0.06) $\mu \mathrm{mol} / 1$, respectively). Serum retinol concentrations decrease as part of an inflammatory response even in vitamin A-sufficient individuals, which is a physiological response that confounds the interpretation of associations between vitamin A status and malaria morbidity ${ }^{(24)}$. Vitamin A plays an essential role in the proper functioning of the immune system, and is believed to be necessary for host resistance to malaria. Significantly lower vitamin A was observed in other studies on severe malaria when compared with the levels in mild/moderate malaria $^{(6,25)}$, which has been explained by the vitamin A redistribution that takes place in the extravascular spaces to allow increased bioavailability to the tissues ${ }^{(9)}$. In addition, the synthesis of acute phase reactants may increase the need for retinol uptake, since it may help incorporate mannose into glycoprotein during synthesis ${ }^{(37)}$.

Plasma albumin, $\mathrm{Ca}$ and $\mathrm{Mg}$ levels have also been found to be particularly depleted with disease severity, as we obtained significant $P$ values after intergroup comparisons for albumin and $\mathrm{Mg}(0.0082$ and 0.0064 , respectively). Albumin is considered a negative acute phase protein (i.e. its level decreases as a consequence of the acute phase response) ${ }^{(38)}$. It is known as a lipid carrier protein in blood, and it has been shown that both lipids and serum albumin are essential for optimum parasite growth in vitro ${ }^{(7,15)}$.

Since plasma $\mathrm{Ca}$ is known to vary with albumin concentrations $^{(39)}$, we calculated correlations between $\mathrm{Ca}$ and albumin values. A positive and significant correlation was found only in the control subjects. This is in line with previous studies showing statistically significant correlations between $\mathrm{Ca}$ and albumin in normal subjects ${ }^{(40,41)}$. Correlations were not statistically significant in all the malaria patient groups. Malaria is characterised by the destruction of erythrocytes due to parasite multiplication ${ }^{(2,3)}$. This haemolysis leads to the release of the intracellular $\mathrm{Ca}$ into the plasma $^{(10)}$, and could participate in an increase in the plasma $\mathrm{Ca}$. On the other hand, albumin was lower in the malaria groups than in the control group. However, as shown in Table 3, mean Ca values were low in all the patient groups. Several studies have reported the reliance of $P$. falciparum for $\mathrm{Ca}$ for the regulation of its cell cycle and for its long-term survival $^{(42,43)}$.
From the analysis of data of the only six fatal cases, low retinol and albumin deficiencies as well as low HDL:LDL cholesterol ratio could be seen as indicators of poor prognosis. The mean HDL:LDL ratio was $0 \cdot 50$ (SE 0.19), which was very near to the lower limit of the normal range of $0 \cdot 4$. However, the mean WAZ in these fatal cases was -0.80. Despite the low number of fatal cases, these data suggest that generally patients with $\mathrm{CM}$ who died were not suffering from severe undernutrition on admission, and therefore, it is unlikely that undernutrition was a primary factor for their poor outcome.

Overall, the present study shows a remarkably high prevalence of severe and moderate undernutritions in children with MA, but not in children with $\mathrm{CM}$ alone. An overall perturbation of the lipid profile in children with severe malaria in general and in children with CM in particular is also detected, which is consistent with a large number of literatures showing decreased cholesterol and increased TAG in acute infection. Still, the values remained in their normal ranges. Also, low plasma retinol was found in children with severe malaria than in controls or children with UCM. Plasma albumin, $\mathrm{Ca}$ and $\mathrm{Mg}$ levels are also low in the severe malaria patients. Low retinol and albumin concentrations and low HDL:LDL cholesterol ratios appear to be indicators of poor prognosis in patients with $\mathrm{CM}$; however, these children were not suffering from undernutrition on admission. Even though malnutrition may increase the risk of malaria, it did not appear to be a primary factor associated with poor outcome. Further studies are needed to look more closely into the association between malnutrition and malaria, taking into account acute phase status, as indicated by acute phase protein concentrations (such as $\mathrm{C}$-reactive protein) in the process leading to severe malaria.

\section{Acknowledgements}

The present work was supported by the European Commission under the FP6 framework programme, Call number 3; Project number: LSHP-CT-2006-037749. No conflict of interest has been reported. The authors' contributions are as follows: J. B. P. M. contributed significantly to the in-hospital patient recruitments, laboratory analysis and the drafting of the manuscript; I. G. contributed to the design of the study, laboratory analysis and the drafting of the manuscript; H. F. K. contributed to the in-hospital recruitments of patients and the laboratory analysis; O. Z. contributed to the in-hospital recruitments of patients; V. C., G. E. R. G and P. H. A. Z. contributed to the design of the study.

\section{References}

1. Snow RW, Guerra CA, Noor AM, et al. (2005) The global distribution of clinical episodes of Plasmodium falciparum malaria. Nature 434, 214-217.

2. Idro R, Jenkins NE \& Newton CRJC (2005) Pathogenesis, clinical features, and neurological outcome of cerebral malaria. Lancet Neurol 4, 827-840.

3. Biemba G, Dolmans D, Thuma PE, et al. (2000) Severe anaemia in Zambian children with Plasmodium falciparum malaria. Trop Med Int Health 5, 9-16.

4. Walker SP, Wachs TD, Gardner JM, et al. (2007) Child development: risk factors for adverse outcomes in developing countries. Lancet 369, 145-157. 
5. Field CJ, Johnson IR \& Schley PD (2002) Nutrients and their role in host resistance to infection. J Leukoc Biol 71, 16-32.

6. Gouado I, Lehman LG, Mbouyap Y, et al. (2007) Influence of malaria on the serum levels of vitamin A, zinc and calcium of children in Douala, Cameroon. Afr J Biotechnol 6, 871-876.

7. Akpotuzor JO, Udoh AE \& Etukudo MH (2007) Total antioxidant status, vitamin A, C and carotene levels of children with $P$. falciparum infection in University of Calabar Teaching Hospital (UCTH), Calabar. Pak J Nutr 6, 485-489.

8. Cooper R, Labadarias D \& Louw ME (2002) Serum vitamin A and $\mathrm{E}$ concentrations in acute falciparum malaria; modulators or markers of severity. Am J Clin Nutr 53, 84-96.

9. Thurnham DI (1996) Antioxidants and proxidants in malnourished populations. Proc Nutr Soc 49, 173-185.

10. Maitland K, Pamba A, Fegan G, et al. (2005) Perturbations in electrolytes levels in Kenyan children with severe malaria complicated by acidosis. Clin Infect Dis 40, 9-16.

11. Mitamura T, Hanad K, Ko-Mitamura P, et al. (2000) Serum factors governing intraerythocytic development and cell cycle progression of Plasmodium falciparum. Parasitol Int 49, 219-229.

12. Nilsson-Ehle I \& Nilsson-Ehle P (1990) Changes in plasma lipoproteins in acute malaria. $J$ Int Med 227, 151-155.

13. Davis TME, Sturm M, Zhang YR, et al. (1993) Platelet-activating factor and lipid metabolism in acute malaria. J Infect $\mathbf{2 6}$, 279-285.

14. Brotons C, Ribera A, Perich RM, et al. (1997) Worldwide distribution of blood lipids and lipoproteins in childhood and adolescence: a review study. Atherosclerosis 139, 1-9.

15. El-Tahir AE, Malhotra P \& Chauhan VS (2003) Uptake of proteins and degradation of human serum albumin by Plasmodium falciparum - infected human erythrocytes. Malaria J 2, 1-8.

16. Mohanty S, Mishra SK, Das BS, et al. (1992) Altered plasma lipid pattern in patients with $P$. falciparum malaria. Ann Trop Med Parasitol 86, 601-606.

17. Faucher JF, Ngou-Milama E, Missinou MA, et al. (2002) The impact of malaria on common lipid parameters. Parasitol Res 88, 1040-1043.

18. Bentz MH \& Magnette $\mathbf{J}$ (1998) Hypocholesterolemia in the acute phase of inflammation during sepsis. Rev Med Int 19, 168-172.

19. Bansal D, Bhatti HS \& Sehgal R (2005) Role of cholesterol in parasitic diseases. Lipids Health Dis 4, 10.

20. WHO (2000) Severe falciparum malaria. Trans $R$ Soc Trop Med Hyg 94, 1-90.

21. Pankoui MJB, Gouado I, Fotso KH, et al. (2008) Clinical presentation, haematological indices and management of children with severe and uncomplicated malaria in Douala, Cameroon. Pak J Biol Sci 11, 2401-2406.

22. Molyneux ME, Taylor TE, Wirima JJ, et al. (1989) Clinical features and prognostic indicators in paediatric cerebral malaria: a study of 131 comatose Malawian children. Q J Clin Pathol 3, $441-459$

23. Jeremiah ZA \& Uko EK (2007) Comparative analysis of malaria parasite density using actual and assumed white blood cell counts. Ann Trop Paediatr 27, 75-79.

24. Caufield LE, Richard SA \& Black RE (2004) Undernutrition as an underlying cause of malaria morbidity and mortality in children less than five years old. Am J Trop Med Hyg 71, $55-63$.

25. Dogan Y, Erkan T, Yalvac S, et al. (2005) Nutritional status of patients hospitalized in paediatric clinic. Turk $J$ Gastroenterol 16, 212-216.

26. Bieri J, Teresa G, Tolliver BS, et al. (1979) Simultaneous determination of tocopherol and retinol in plasma or red cells by high pressure liquid chromatography. Am J Clin Nutr 3, $2143-2149$

27. Johnson R, McNutt P, MacMahon S, et al. (1997) Use of the Friedewald formula to estimate LDL-cholesterol in patients with chronic renal failure on dialysis. Clin Chem $\mathbf{4 3}$, $2183-2184$

28. Schellenberg D, Schellenberg JR, Mushi A, et al. (2003) The silent burden of anaemia in Tanzanian children: a communitybased study. Bull World Health Organ 81, 581-590.

29. Schaible UE \& Kaufmann SHE (2007) Malnutrition and infection: complex mechanisms and global impacts. PLoS Med 4, 806-810.

30. Reibnegger G, Fuchs D \& Hausen A (1987) The dependence of cell-mediated immune activation in malaria on age and endemicity. Trans $R$ Soc Trop Med Hyg 81, 729-733.

31. Stephensen CB (1999) Burden of infection on growth failure. $J$ Nutr 129, 534S-538S.

32. Shankar AH (2000) Nutritional modulation of malaria morbidity and mortality. J Inf Dis 182, S37-S53.

33. Djoumessi S (1989) Serum lipids and lipoproteins during malaria infection. Pathol Biol 37, 909-911.

34. Jacobs DR Jr, Hebert B, Schreiner PJ, et al. (1997) Reduced cholesterol is associated with recent minor illness: the CARDIA Study. Coronary Artery Risk Development in Young Adults. Am J Epidemiol 146, 558-564.

35. Mohanty S, Mishra SK, Das BS, et al. (1992) Altered plasma lipid pattern in patients with P. falciparum malaria. Ann Trop Med Parasitol 86, 601-606.

36. Reibnegger G, Fuchs D \& Hausen A (1987) The dependence of cell-mediated immune activation in malaria on age and endemicity. Trans $R$ Soc Trop Med Hyg 81, 729-733.

37. Filteau SM (1999) Vitamin A and the acute-phase response. Nutrition 15, 326-328.

38. Goselle ON, Onwuliri COE \& Onwuliri VA (2007) Malaria and the effect of malaria parasitaemia on albumin level among HIV/ AIDS patients in Jos, Nigeria. J Med Sci 7, 1187-1191.

39. Butler SJ, Payne RB, Gunn IR, et al. (1984) Correlation between serum ionised calcium and serum albumin concentrations in two hospital populations. Br Med J 289, 948-950.

40. Payne RB, Little AJ, Williams RB, et al. (1973) Interpretation of serum calcium in patients with abnormal serum protein. Br Med J 4, 643-646.

41. Ogunkolo OF, Amballi AA, Adenaike FA, et al. (2006) Derivation of a formula for adjusting the total serum calcium in Nigeria environment. Afr J Biotechnol 5, 1250-1253.

42. Garcia CR (1999) Calcium homeostasis and signalling in the blood-stage malaria parasite. Parasitol Today 15, 488-491.

43. Gazarini M, Thomas A, Pozzan T, et al. (2003) Calcium signalling in a low calcium environment: how the intracellular malaria parasite solves the problem. J Cell Biol 161, 103-110. 